\title{
Imaging X-ray Polarimeter for Solar Flares (IXPS)
}

\author{
Michael Hosack . J. Kevin Black . \\ Philip Deines-Jones - Brian R. Dennis . \\ Joanne E. Hill - Keith Jahoda - Albert Y. Shih . \\ Christian E. Urba * A. Gordon Emslie
}

Received: date / Accepted: date

\begin{abstract}
We describe the design of a balloon-borne Imaging X-ray Polarimeter for Solar flares (IXPS). This novel instrument, a Time Projection Chamber (TPC) for photoelectric polarimetry, will be capable of measuring polarization at the few percent level in the 20-50 keV energy range during an M- or X-class flare, and will provide imaging information at the $\sim 10$ arcsec level. The primary objective of such observations is to determine the directivity of nonthermal high-energy electrons producing solar hard X-rays, and hence to learn about the particle acceleration and energy release processes in solar flares. Secondary objectives include the separation of the thermal and nonthermal components of the flare X-ray emissions and the separation of photospheric albedo fluxes from direct emissions.
\end{abstract}

Keywords Solar flare $\cdot$ X-ray polarimetry $\cdot$ Particle tracking $\cdot$ GEM $\cdot$ TPC

M. Hosack - B. R. Dennis - A. Y. Shih

Code 671, NASA's Goddard Space Flight Center, Greenbelt, MD 20771, USA

E-mail: brian.r.dennis@nasa.gov

M. Hosack

ADNET Systems, Inc., 164 Rollins Avenuc, Suite 303, Rockville, MD 20852, USA

J. K. Black

Rock Creek Scientific, 1200 East-West Hwy, Suite 1411, Silver Spring, MD 20910, USA

P. Deines-Jones - J. E. Hill - K. Jahoda

Code 662, NASA's Goddard Space Flight Center, Greenbelt, MD 20771, USA

J. E. Hill - C. E. Urba

CRESST/Universities Space Research Association, 10211 Wincopin Circle, Suite 500, Columbia, MD 21044, USA

A. G. Emslic

Department of Physics \& Astronomy, Western Kentucky University, Bowling Green, KY 42101, USA 


\section{Introduction}

Astrophysical interest in high-energy polarization measurements has remained high for decades. The initial measurements of the X-ray polarization of the Crab Nebula were made in the 1970s $[39,57]$ but polarization measurements have remained instrumentally challenging. Only recently have the measurements of the Crab been extended to gamma-ray energies $[13,18]$. Observationally, however, the field is poised to explode as is evident from the recent selection of the Gravity and Extreme Magnetism SMEX (GEMS) [50]. The workshop on "The Coming Age of X-ray Polarimetry" held in Rome in April 2009 attracted over 100 participants and featured many presentations on now feasible instruments (in various stages of development) with sensitivities sufficiently high to be of interest for astrophysics [3].

Our understanding of electron acceleration in solar flares remains limited despite detailed spectral and temporal imaging of the resulting hard X-ray (HXR) bremsstrahlung. Polarization measurements provide direct information on the degree of beaming of the accelerated electrons, an important aspect of particle acceleration models. Available measurements are tantalizing but insufficient to distinguish between competing models.

The relatively new application of the time-projection technique to photoelectric polarimetry has emerged in the last few years and provides important new capabilities in the $20-100 \mathrm{keV}$ band. This is an important complement to Compton polarimeters, which remain more sensitive at energies above $100 \mathrm{keV}$. This photoelectric technique readily scales to the large effective areas required for solar HXR imaging polarimetry.

\subsection{Scientific Motivation}

Our knowledge of the hard X-ray (HXR) bremsstrahlung produced in solar flares, a key diagnostic of electron acceleration, remains fundamentally incomplete. The temporal and spectral signatures have been studied for almost half a century, culminating in the unprecedented observations from the Reuven Ramaty High-Energy Solar Spectroscopic Imager (RHESSI) [30]. We now have detailed knowledge of the spectrum and the spatial structure of the HXR sources and their evolution with time. Kontar and Brown [25] have presented a model including spectral energy contributions from both direct emission and the albedo flux, caused by Compton scatter of downward-propagating photons in the solar photosphere, with a resulting enhancement of flux directed toward the observer [2]. They applied this model to spectra from two flares observed by RHESSI enabling a determination of the directivity (i.e., degree of anisotropy) of the the nonthermal electron distribution for each flare. Polarimetry would provide more powerful measures of the directivity than relying on energy spectra alone. Polarimetry yields direct information on the directivity of the emitting electron population and so provides critical constraints on the acceleration mechanism. Imaging polarimetry is necessary to separate the flare footpoints and coronal sources since they are likely to have vastly different degrees of polarization. Imaging polarimetry can also reveal the characteristic polarimetric signature of the photospheric albedo patch, clearly distinguishing it from the direct X-ray flux, which 
is expected to have a significantly different spatial distribution of the magnitude and orientation of the polarization vector [2]. Such measurements are especially significant in the 10-100 keV range, where the fractional contribution of the reflected albedo flux to the total measured flux is the greatest-up to $50 \%$ at $30 \mathrm{keV}[2,26]$. However, there are no convincing measurements of HXR polarization.

We are finally seeing the onset of observations capable of testing the numerous theoretical predictions of solar HXR polarization that have been in the literature for decades. The few existing polarization measurements are intriguing but inconclusive. Collectively, they suggest that the magnitude of the polarization vector is of the order predicted by models that have a strong anisotropy of the emitting electrons. However, the measured orientation of this vector may be in a direction substantially different from the local solar radial that is predicted by most solar flare models [14]. This raises the fascinating possibility that significant refinements may be required in our models for particle acceleration and transport in solar flares.

The cross-section for bremsstrahlung emission depends on the linear polarization angle of the emitted photon measured relative to the plane containing the pre-collision electron velocity vector and the direction of photon emission [19]. Taking into account that the accelerated electrons spiral around guiding magnetic field lines, one customarily measures the polarization angle resulting from a given electron collision with respect to a fixed plane defined by two vectors: the guiding magnetic field and the direction of photon emission (i.e., the line-of-sight to the observer) [20]. If the magnetic field direction is taken to be the solar vertical, this reference plane contains the vector from the center of the solar disk to the flare location. By convention, a polarization fraction is denoted as positive if the orientation of the polarization vector is perpendicular to this radial vector in the solar disk. The polarization fraction is negative if the polarization vector is parallel to the radial vector.

Numerous authors have constructed a variety of solar flare models to calculate the expected polarization degree and orientation. They have incorporated different source geometries, electron energy spectra, and angular distributions. They have invoked propagation of nonthermal electrons into a thick target $[11,21,27,2,28,29]$, emission from a thermal source with a nonuniform temperature structure [15], and a combination of the above [16]. Models involving the downward beaming of accelerated electrons generally predict a predominantly radial (negative) direction for the polarization vector in the deka-keV photon energy range. Magnitudes range from a few percent to a few tens of percent, depending mostly on the assumed degree of downward anisotropy of the emitting electrons. A non-radial polarization vector is predicted for an extended thermal source [15], in which the anisotropy of the electron distribution is driven by the temperature gradients in the extended source. Of course, the scattering of the accelerated electrons as they move throughout the target atmosphere causes the angular distribution of the accelerated electrons, as well as their HXR polarization signal, to vary from point to point [29]. Thus, measuring the polarization at different spatial locations in the flare provides a much more discriminating diagnostic on the anisotropy of the accelerated electrons, emphasizing the importance of imaging polarimetry.

Measurement of solar flare hard X-ray polarization began in the 1970s with a series of measurements from the Russian Intercosmos satellites [51,52,53,54]. How- 
ever, the reliability of these pioneering measurements has been questioned [12]. Attempts to measure solar HXR polarization from a Space Shuttle payload were similarly inconclusive due to contamination of the lithium scatterer [55].

RHESSI is capable of limited non-imaging polarimetry thanks to a small beryllium scatterer placed in the detector tray [33]. Solar X-ray photons Compton scatter off this target into the rear segments of the four nearest-neighbor germanium detectors, and the relative count rates in these four detectors provide information on the polarization. A preliminary polarization signal has been reported [34] from the RHESSI observation of the GOES class X4.8 flare of 2002 July 23, a flare located near the east limb that exhibited many of the characteristics associated with nonthermal electron acceleration and propagation. These include a hard power-law photon spectrum [23] and bright hard X-ray footpoints [17]. The degree of polarization was measured to be $(15 \pm 4) \%$ at an angle of $(79 \pm 4)^{\circ}$ counterclockwise from the solar west, corresponding to an angle $\sim 64^{\circ}$ to the local solar radial direction. However, strong and consistent gamma-ray line redshifts in this near-limb event were reported [38], indicating that the magnetic field guiding the trajectories of the emitting particles might be significantly non-vertical. This could indeed plausibly account for the measured orientation of the polarization vector [14]. Unfortunately, these polarization results remain questionable because of additional unexplained systematic variations in the counting rates of the four detectors nearest the beryllium scatterer as the spacecraft rotates.

RHESSI's Ge detectors are also active Compton scatterers at higher photon energies ( $>200 \mathrm{keV}$ ), and correlation of coincident counts in adjacent detectors can be used to infer the incident polarization. Marginal gamma-ray polarizations have been reported at photon energies in the range $0.2-1.0 \mathrm{MeV}$ in two flares measured from such detector-detector correlations [9]. In one event on 2003 October 28 , the polarization vector was reported to be approximately radial with a magnitude of $(11 \pm 5) \%$. In the other event on 2002 July 23 , the polarization vector was approximately transverse with a magnitude weakly constrained to lie between $6 \%$ and $48 \%$. Also using the detector-to-detector scattering method, Suarez-Garcia et al. [48] analyzed RHESSI data for seven near-limb flares in the energy range from $100-350 \mathrm{keV}$. They found polarization magnitudes ranging from $2 \%$ to $54 \%$ but with large $(1 \sigma)$ error bars of order $20-30 \%$. The orientations of the polarization vectors relative to the radial direction ranged from $35^{\circ}$ to $85^{\circ}$. Comparison of the ensemble data with various theoretical predictions allowed rejection of a high-pitch-angle injection model (model 5 of [28]), but were consistent both with other models $[28,2]$ and with $0 \%$ polarization.

Extremely large polarization magnitudes of $50-70 \%$ for the X 10 flare of $2003 \mathrm{Oc}$ tober 29 have been reported using $20-100 \mathrm{keV}$ data from the CORONAS-F SPR-N hard X-ray polarimeter $[10,61]$. This instrument records photons scattered from a series of beryllium plates into a set of six scintillation detectors. Such a polarization magnitude is extremely high, especially considering the near-disk-center position (S15W02) of this event. It is far larger than predicted by any model hitherto proposed and indeed is at the limit of what can be expected from a model with $100 \%$ anisotropy in the emitting electron population. Further, the orientation of the polarization plane, determined within a claimed uncertainty of $30^{\circ}$, was parallel to the solar equator, representing a near-azimuthal polarization vector. Both the magnitude and orienta- 
tion of such a polarization vector imply a highly non-vertical guiding magnetic field. Even more remarkable is that out of 90 flares studied, only 25 had a sufficiently high polarization to be detected, and only three had polarization values as high as those exhibited by the October 29 event [61]. Nevertheless, a confirmation of this very high polarization signal would be a powerful constraint on flare models.

In summary, observations to date are highly inconclusive, in no small part because they have been made using instruments that were not optimized or calibrated for the measurement of polarization. With the increasing realization that polarization measurements provide uniquely insightful information on the physical processes at work in particle acceleration sites throughout astrophysics, the time is propitious to develop an instrument specifically tailored to the measurement of solar hard X-ray polarization. The energy range from $\sim 10$ to $100 \mathrm{keV}$ is particularly pertinent since it is not only where the polarized component of the nonthermal flare emission is the brightest, it is also where there is a significant contribution from the highest temperature thermal sources and where photospheric albedo makes its maximum contribution [2, 26]. Thus, sensitive and reliable polarization measurements will constitute a quantum leap in capability that can resolve many of the current ambiguities and outstanding questions in solar flare physics.

\subsection{Instrument Requirements}

The instrumental requirements are driven by our goal of making imaging $\mathrm{X}$-ray polarization measurements of the nonthermal HXR emissions from solar flares at balloon altitudes. The primary instrumental requirements are thus driven by the required polarization sensitivity and imaging capability in the $20-100 \mathrm{keV}$ energy range. Since there are, on average, one or two M1 or brighter flares per week at solar maximum, an instrument must be able to measure polarization in M-class flares to have a high probability of success in a single long-duration balloon flight. Our survey of observational and theoretical literature suggests that a detection limit of better than $10 \%$ is sufficient to make meaningful measurements. Also, imaging at the $\sim 10$ arcsec level is key to distinguishing the polarization signal from different parts of the flare: e.g., the footpoints, the loop tops, and the albedo patches.

Our instrument concept meets the balloon mission requirements by having sufficiently large area, high quantum efficiency, and high polarization sensitivity to be a suitable detector in the $20-50 \mathrm{keV}$ band. The imaging capability is provided by $\mathrm{X}$ ray modulation collimators in front of the detector. Figure 1 shows the effective area of a conceptual design that would require four Time Projection Chambers (TPCs), each with a sensitive area of $10 \times 10 \mathrm{~cm}^{2}$ and depth of $30 \mathrm{~cm}$. On orbit, this instrument would measure polarization in many brighter flares from $<20 \mathrm{keV}$ to $>50 \mathrm{keV}$, overlapping the energy range above $\sim 50 \mathrm{keV}$ covered with instruments using Compton scattering like the Gamma Ray Polarimeter Experiment (GRAPE) [35] and the Gamma-Ray Imaging/Polarimeter for Solar Flares (GRIPS) [44]. 


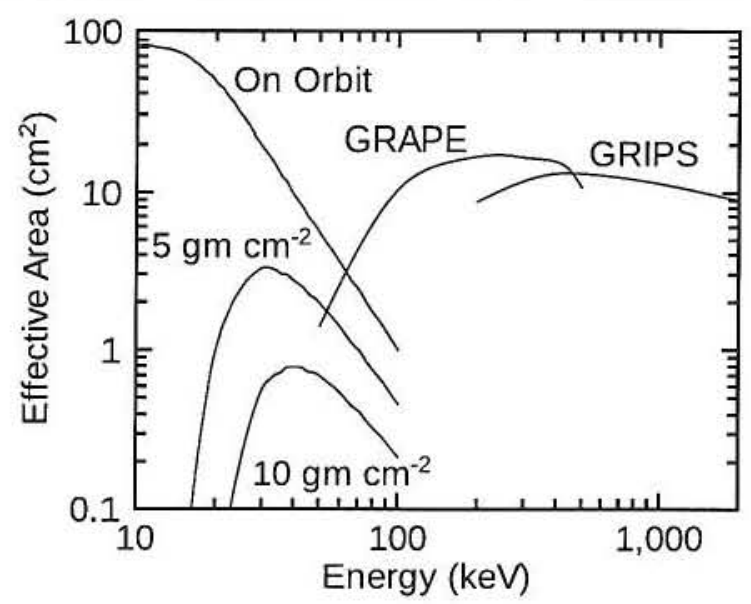

Fig. 1 The effective area of our TPC photoelectric polarimeter concept in low-Earth orbit and at balloon altitude with two different atmospheric path lengths. The total effective area of the 36 GRAPE modules planned for a 2013 balloon flight in Antarctica is shown for comparison [35]. Also shown is the effective area of GRIPS with its final complement of detectors (personal communication with Albert Shih). Atmospheric attenuation for a path length of $10 \mathrm{gm} \mathrm{cm}^{-2}$ is included in the GRIPS and GRAPE curves shown. The GRAPE array will be uncollimated for the 2013 flight and GRIPS has a 50\% transmitting grid for imaging, whereas our TPC polarimeter concept includes a $25 \%$ transmitting collimator for imaging. These collimation transmission factors are included in the curves shown.

\section{Technical Approach}

In the following sections, we describe photoelectric time projection polarimeters based on gas micropattern proportional counters, and provide details of our prototype detector module. This technology is also the basis of the X-ray Polarimeter Instrument (XPI) on GEMS [50] and the Gamma-Ray Burst Polarimeter (GRBP) [22]. IXPS will use a different gas, a larger volume, and higher pressure than used for GEMS-XPI or GRBP to achieve better sensitivity at higher energies. Its design leverages off our recent and ongoing work in soft X-ray polarimetry and micropattern detector technology $[6,5,7,22]$.

\subsection{Photoelectric Polarimetry}

In the X-ray energy range of interest for polarization measurements of solar flares (above $\sim 20 \mathrm{keV}$ ), photons can interact by two physical processes: photoelectric absorption and Compton scattering. In both processes, the trajectory of the observed particle is correlated to the direction of the electric-field vector of the incident photon with a polarization-dependent angular distribution modulated as $\cos ^{2} \psi \cdot \psi$ is defined somewhat differently for the two processes. For Compton scattering, $\psi$ is the azimuth angle measured from perpendicular to the direction of the electric-field vector of the incident photon to the direction of the emitted (observed) photon. For photoelectric absorption, $\psi$ is the azimuth angle measured from the direction of the electric-field 
vector of the incident photon to the direction of the emitted (observed) photoelectron. The amplitude and phase of this modulation provides a measure of the amplitude and phase of the X-ray linear polarization. The instrumental challenge is to measure the azimuthal direction of the photoelectron or the Compton-scattered photon.

Compton scattering becomes favored at energies above about $50 \mathrm{keV}$ as the Compton cross-section begins to dominate the photoelectric cross-section in common detector materials. Solar X-ray polarimeters flown so far, RHESSI being the latest example, have used Compton scattering with a passive low-Z scatterer surrounded by multiple X-ray detectors. Above $100 \mathrm{keV}$, the technique can be improved by using active scatterers that detect the knock-on electron as in GRAPE [35] or GRIPS [44].

Below $100 \mathrm{keV}$, polarization measurements have recently become practical with the development of a highly sensitive measurement technique based on imaging the photoelectron track using a micropattern gas detector (MPGD) [47]. The sensitivity of MPGD polarimeters can be optimized by implementing a time projection readout scheme [6]. These time-projection chambers (TPCs) have been demonstrated as sensitive polarimeters in the $2-10 \mathrm{keV}$ band but have not yet been optimized for higher energies. The main advantage of a TPC with a one-dimensional strip readout compared to a MPGD with a pixel readout (e.g., [47]) is that charge diffusion is decoupled from interaction depth/quantum efficiency. Also, since the TPC requires only a onedimensional strip readout, it more readily scales to large volumes. Designs for other types of polarimeters exist covering the $10-100 \mathrm{keV}$ range $[47,46]$.

\subsection{Photoelectric Polarimetry with a Time-Projection Chamber (TPC)}

As a result of photoelectric absorption, a gas atom ejects a photoelectron preferentially in the direction of the incident photon electric field with a $\sin ^{2} \theta \cos ^{2} \phi$ probability distribution, where $\theta$ is the polar angle and $\phi$ is the polarization sensitive azimuthal angle. In the active volume of a TPC, this photoelectron leaves a trail of ionization along its path as it loses energy and multiple Coulomb scatters in the gas. The charges drift towards a Gas Electron Multiplier (GEM) ${ }^{1}$ under the influence of a uniform electric field (Fig. 2).

At the detection plane, electron avalanches in the GEM amplify the primary ionization and it is collected on the readout strips. The strips are individually instrumented with continuously sampling analog-to-digital converters (ADCs) to measure both the amount of charge and its arrival time (Fig. 2, right). Position information normal to the strips between the GEM and the drift electrode is determined by the arrival time of individual clusters of primary ionization. The numbers of the hit strips and the arrival times then allow a track image to be determined as projected onto the $\mathrm{XY}$ plane as indicated, with the size of the dots being proportional to the amount of charge. The emission direction of each photoelectron is then estimated from the track image. Thus, this photoelectric polarimeter is simultaneously sensitive to all phases of polarization and does not require rotation about the $\mathrm{Z}$-axis. Of course, rotation

1 GEMs and GEMS are distinct acronyms. GEMs (Gas Electron Multipliers) are detector clements; GEMS (Gravity and Extreme Magnetism SMEX) is a NASA Explorer mission [50]. 


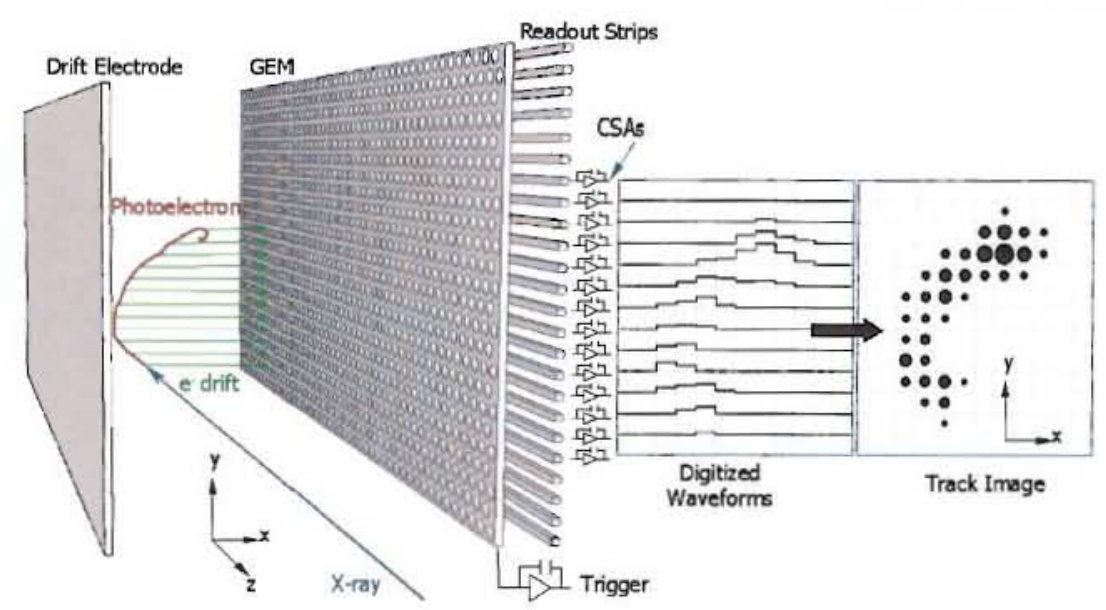

Fig. 2 Illustration of the operation of a time projection chamber (TPC). An X-ray photon enters the gas volume as shown along the $-\mathrm{Z}$ axis and suffers a photoclectric interaction. The resulting photoclectron travels along an irregular path as it is scattered in multiple Coulomb collisions. The resulting trail of ionization drifts relatively slowly under the influence a strong electric field between the GEM and the drift electrode. The signals are read out on multiple strips through charge-sensitive amplifiers (CSAs). The digitized waveforms on each strip reveal the track of the photoelectron projected onto the $\mathrm{XY}$ plane. The time projection polarimeter uses a simple strip readout to form pixelized images of photoclectron tracks. It forms an image by digitizing the signal on each anode strip. Signals proportional to the charge deposited on each strip are shown on the left. The resulting image on right shows the interaction point, emission anglc, and end of the track. Each circle has a size proportional to the deposited charge in each virtual pixcl. In this example, the circles are on a 130 micron spacing.

about the Z-axis would be desirable to allow the orthogonal distance scales to be more accurately normalized and systematic effects to be evaluated.

The electron track images in a TPC are self-contained so that the emission angle can be reconstructed without a priori knowledge of the interaction point. Therefore, azimuthal symmetry around the line of sight is not required to avoid false modulation. This also allows the analysis parameters to be optimized after the fact, and provides for the rejection of background and charged particle events.

The FWHM energy resolution of this type of TPC detector is $\sim 0.5 E^{0.5} \mathrm{keV}$, where $E$ is the $\mathrm{X}$-ray photon energy in $\mathrm{keV}$. This corresponds to $\sim 2 \mathrm{keV}$ at $20 \mathrm{keV}$ and $\sim 3.5 \mathrm{keV}$ at $50 \mathrm{keV}$, similar to that of solid-state detectors envisioned for Compton-scatter polarimeters [35]. This high-resolution spectroscopy is a significant advantage in handling narrow lines in the background spectrum and in measuring the often very steep thermal solar flare spectra with an equivalent power-law spectral index $\gamma$ of $>7$ and a potentially sharp break to the flatter nonthermal component at higher energies.

Time-projection polarimetry is a technique (Fig. 2) that readily scales to the large effective areas needed for solar imaging polarimetry using modulation collimators. While time-projection polarimeters in the $2-10 \mathrm{keV}$ band are well established $[7,22]$, they have not previously been applied to hard X-ray polarimetry. A TPC polarimeter with bi-grid modulation collimators for imaging would provide a powerful tool for 


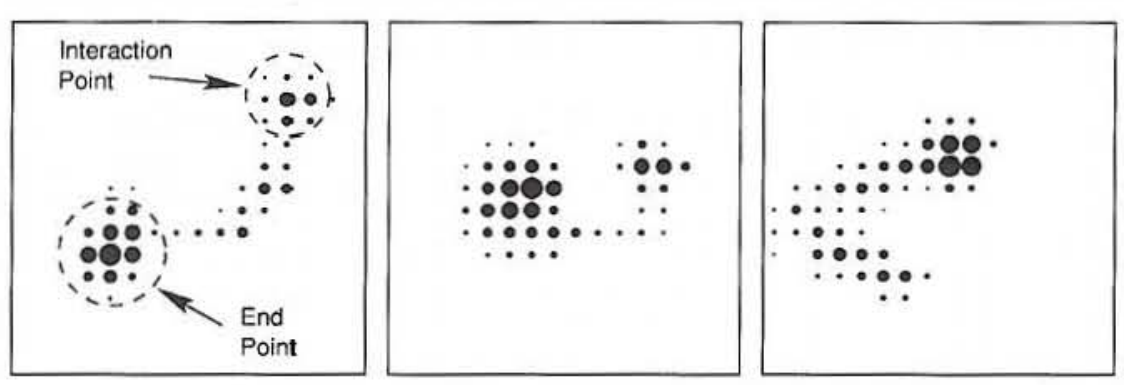

Fig. 3 Example tracks of photoclectrons from $6 \mathrm{keV}$ X-rays. Note that the fainter end of a track indicates the location of the photoelectric interaction while the brightest pixels reveal the end point of the electron path.

solar flare investigations by combining large effective area, low systematic errors, low background, and good energy resolution.

In our test chamber, images of the photoelectron tracks from $6 \mathrm{keV}$ X-rays are formed as an array of $24 \times 24$ pixels centered on the event barycenter. Typical examples are shown in Fig. 3. Note that the angular information is least affected by scattering near the interaction point [41]. The two-stage moments based algorithm used to estimate the emission angle from the image is described in [7].

Histograms of the emission angles are then fit to the expected functional form: $N(\phi)=A+B \cos ^{2}\left(\phi-\phi_{0}\right)$, where $\phi_{0}$ is the angle of the plane of polarization. The sensitivity to polarization is defined by the modulation, $\mu$, where $\mu=\left(N_{\max }-\right.$ $\left.N_{\min }\right) /\left(N_{\max }+N_{\min }\right)=B /(2 A+B)$ and scales from 0 to 1 . An example of the results from this analysis is shown in Fig. 4.

\subsubsection{Background Rejection}

For any solar polarization measurement, it is important to reject background events from all non-solar X-ray sources. During a large flare, the solar X-ray flux will far exceed the non-solar X-ray background but the charged particle fluxes may be an issue at balloon altitudes.

Three techniques can be used to separate the photoelectron tracks of interest from the tracks of charged particles passing through the TPC.

1. Charged particles will produce straight tracks with relatively uniform density,

2. The readout strips closest to the edges of the chamber (Fig. 2) will be used to define a thin $(2 \mathrm{~mm})$ anticoincidence wall on opposite sides of the TPC. Any track seen in either or both of these two walls will be rejected.

3. Many charged particles will deposit a total energy in the TPC outside our range of interest between 20 and $50 \mathrm{keV}$ and can be rejected on that basis.

\subsubsection{Previous Work}

We have built a large-area prototype TPC polarimeter and have tested it in a variety of gases and at different pressures. It consists of a $10 \times 10 \mathrm{~cm}^{2}$ SciEnergy GEM (shown 

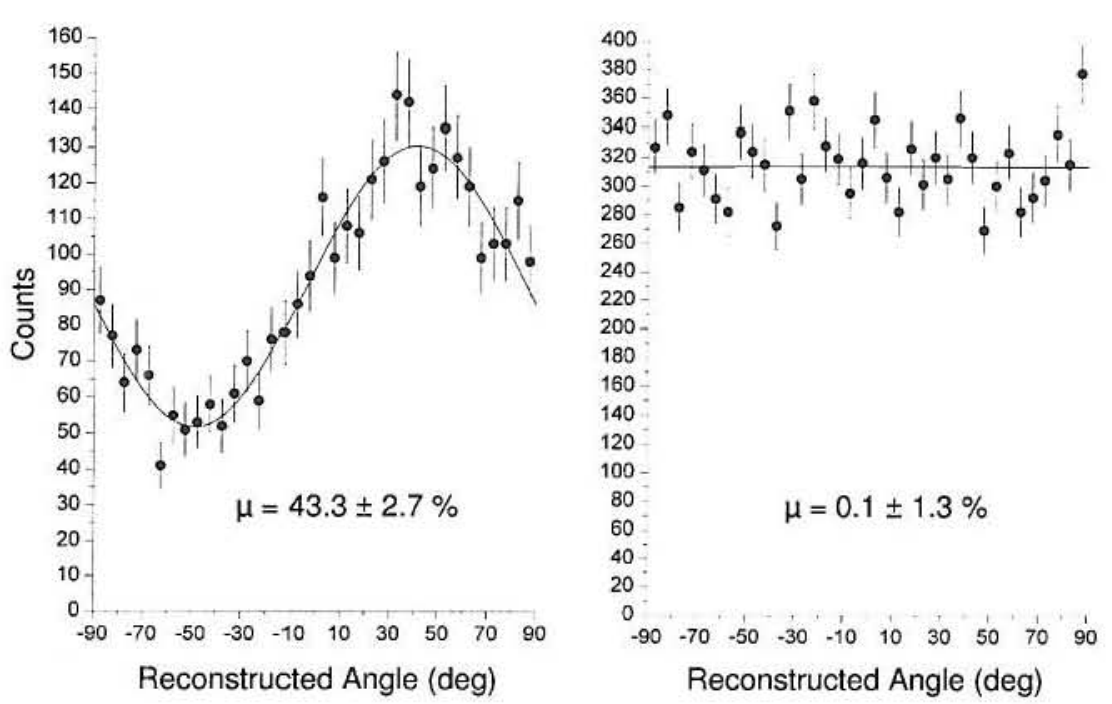

Fig. 4 Reconstructed emission angles for polarized $6.4 \mathrm{keV} X$-rays (left) and unpolarized $5.9 \mathrm{keV} \mathrm{X-rays}$ (right). The lines are fits to the expected functional form, $A+B \cos ^{2} \phi$. The modulation factor, $\mu$, is a measure of the amplitude of the response. The polarized data show a clear response, while the unpolarized data are consistent with no detected signal below the $3 \%$ level. The gas used was $83.3 \% \mathrm{Ne}+8.3 \% \mathrm{CO}_{2}$ $+8.3 \% \mathrm{CS}_{2}$ at $0.63 \mathrm{~atm}$.

in Fig. 5, left) with a $6 \mathrm{~cm}$ drift distance. The GEM is constructed from two $\mathrm{Cu}$ electrodes separated by $100 \mu \mathrm{m}$ of liquid crystal polymer (LCP) insulator with laserdrilled holes in a hexagonal pattern on a $140 \mu \mathrm{m}$ pitch. The readout strips are formed from copper strips on FR4 printed circuit board with a pitch of $121 \mu \mathrm{m}$. However, FR4 outgasses and tends to absorb dimethyl ether (DME) and other gasses that might be used. Thus, for a long duration mission with a sealed chamber, the strips can be changed to be similar to the GEMS X-ray polarimeter instrument (GEMS-XPI) design, where they are on a LCP board with a frame to hold them in place (shown in Fig. 5, right).

The current prototype electronics were built from commercial off-the-shelf components to read out a polarimeter that uses a negative-ion gas, e.g., an argon/carbon disulphide $\left(\mathrm{Ar} / \mathrm{CS}_{2}\right)$ mixture. With such a gas, the photoelectron tracks exhibit lower diffusion and the charge drifts more slowly than in a traditional electron gas, allowing the use of slower, and therefore lower power, electronics. However, detector simulations of the expected X-ray interaction rate from an X-class solar flare indicate that the slow drift velocity in a negative-ion gas results in track confusion at high rates when more than one track occupies a given imaging area during the same readout time. Similarly, high background rates can give rise to significant deadtime in a slow gas as the charge generated takes longer to fully collect in the GEM. These effects are modest for X-rays from a flare or for background rates on a balloon flight over the United States (see Sec. 2.6.1). 


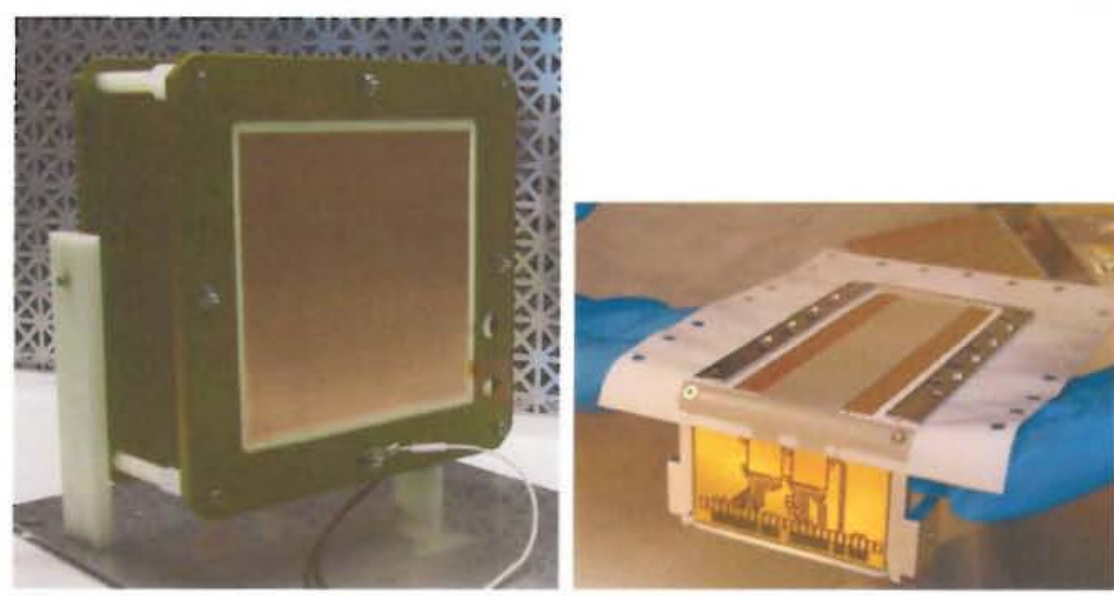

Fig. 5 Left: a $10 \times 10 \mathrm{~cm}^{2}$ SciEnergy GEM (with readout strips removed) and the drift electrode. Right: readout design for GEMS-XPI with an LCP board that could be implemented in the current $10 \times 10 \mathrm{~cm}^{2}$ design to minimize outgassing.

\subsection{Flight Instrument Concept}

A diagram of the overall arrangement of a large orbital instrument is shown in Fig. 6. The TPC shown in this figure is $30 \mathrm{~cm}$ deep requiring three $10 \times 10 \mathrm{~cm}^{2}$ GEM stacks. Stacking GEMs boosts the signal gain by multiplying the charge at each layer. Imaging information is provided by a bi-grid rotating modulation collimator (RMC) with a grid separation of $100 \mathrm{~cm}$. The RMC rapidly modulates the incident X-ray flux to provide information on the spatial Fourier components of the source similar to the RHESSI imaging technique. This rapid modulation requires a fast, electron gas in the TPC.

The concept for a balloon-borne polarimeter requires the following modifications of this basic design. The TPC shown in Fig. 7 is $10 \mathrm{~cm}$ deep, requiring only a single GEM stack. To avoid having to rotate the instrument, this concept uses a fixed bi-grid collimator, still with a grid separation of $\sim 100 \mathrm{~cm}$ (see Sec. 2.5 for more detail). Since this technique does not depend on a rapidly modulating signal, a slow, negative-ion gas can be used in the TPC.

In designing a flight instrument, the basic objective is to achieve a minimum detectable polarization (MDP) of $\sim 10 \%$ for a typical M1 flare. The area of the largest readily-available GEM was assumed, and various fill gasses, drift distances, pitch of readout strips, etc., were considered. Optimum detector parameters were determined by first selecting a gas, then modeling the modulation as a function of X-ray energy, anode pitch, and drift distance.

Choosing the best fill gas requires consideration of drift speed, diffusion, and mean atomic number. A gas with low drift velocity is desirable to achieve high photoelectron spatial resolution with relatively low-speed low-power electronics. A gas with low electron diffusion allows tracks to be resolved over a large drift distance thus enlarging the sensitive area. Increasing the mean atomic number $(\mathrm{Z})$ of the gas 


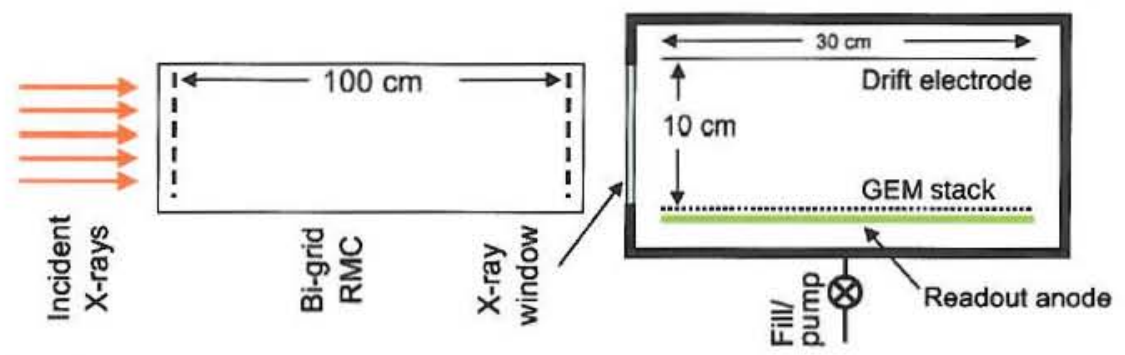

Fig. 6 Overview of a large imaging polarimeter showing the TPC with a $10 \times 10 \mathrm{~cm}^{2}$ aperture and $30 \mathrm{~cm}$ depth requiring three stacks of the largest GEMs now available. Imaging information with $\sim 10$ arcsec resolution would be provided by the bi-grid rotating modulation collimator (RMC) with the front and rear grids separated by $100 \mathrm{~cm}$.

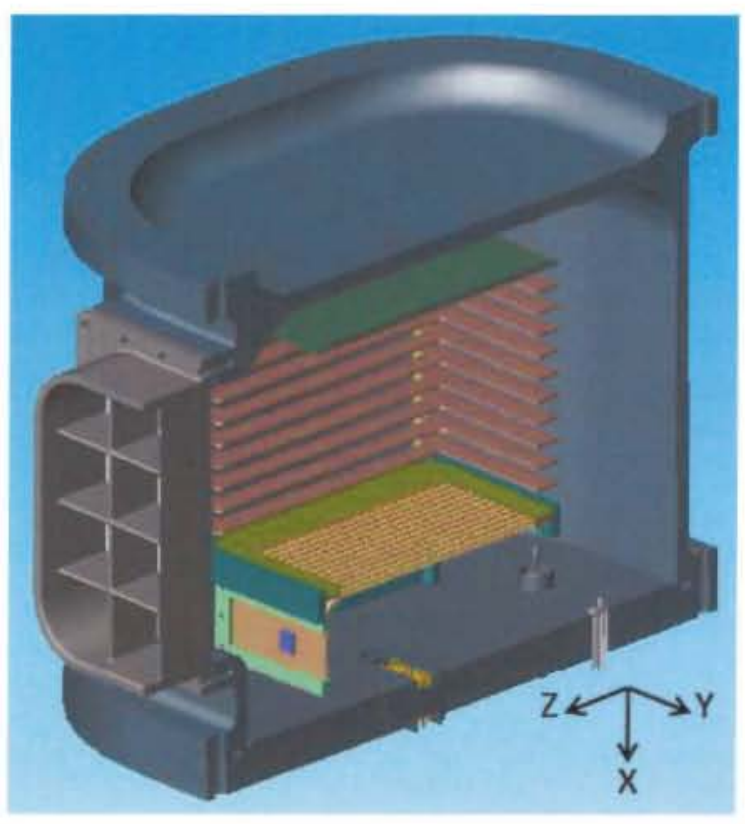

Fig. 7 Cutaway drawing of a single-module TPC using a stack of $10 \times 10 \mathrm{~cm}^{2}$ GEMs (only one GEM shown) with readout strips parallel to the Z-axis. The drift electrode (on top of the shaping electrodes) and the pressure chamber (enclosure) are also shown. Solar X-rays incident from the left near-parallel to the $-\mathrm{Z}$ direction pass through the window shown behind the stiffening supports built to withstand the $3 \mathrm{~atm}$ differential gas pressure.

increases the stopping power and therefore the quantum efficiency of a detector for a given size and gas pressure. But increasing $\mathrm{Z}$ also increases scattering and shortens the usable track extent, making it harder to reconstruct the initial direction of the photoelectron. Furthermore, the incident X-ray energy must be at least twice the Kshell energy of the absorber in order to produce a large modulation. This constraint and increased scattering eliminates xenon-based gases for a $20-50 \mathrm{keV}$ polarimeter, but argon and krypton meet the criteria.

We initially modeled detectors filled with 70:30 Ar:DME (an electron drift gas) for different gas pressures, drift distances, and readout pitches, and selected parameters that maximized polarization sensitivity. The simulations show that the modula- 


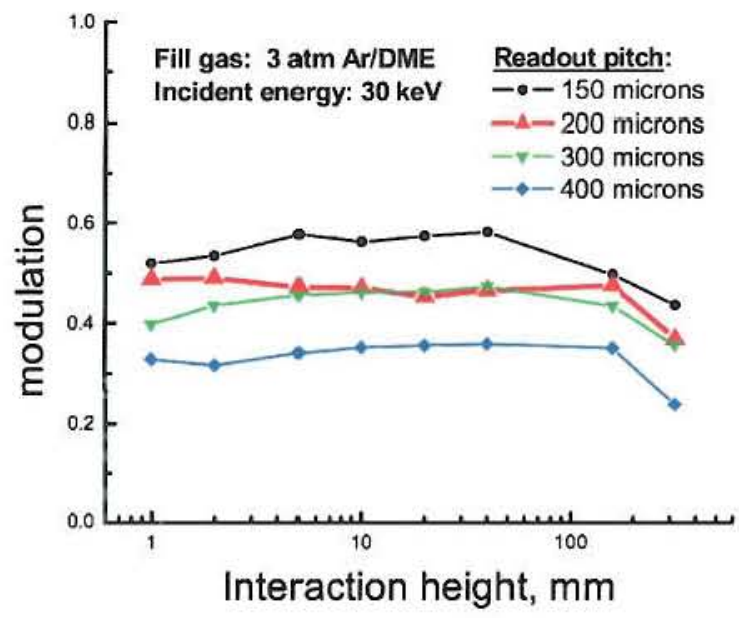

Fig. 8 Simulations at $30 \mathrm{keV}$ showing that the modulation amplitude is approximately constant for a given readout pitch for drift distances (interaction heights) of less than $\sim 200 \mathrm{~mm}$.

tion amplitude at $30 \mathrm{keV}$ is largely independent of drift distances less than $\sim 20 \mathrm{~cm}$ (Fig. 8). Based on this study, we selected a pressure of $3 \mathrm{~atm}$, a maximum drift distance of $10 \mathrm{~cm}$, and a $150 \mu \mathrm{m}$ pitch readout for the flight detector concept. This is within the capabilities of many printed circuit board manufacturers. Owing to its lower diffusion, we expect that a negative-ion drift gas would exhibit a flatter modulation response above $\sim 10 \mathrm{~cm}$, where some drop-off is apparent with Ar:DME.

A negative-ion drift gas would also allow the use of slow and therefore economical low-power readout electronics. Additionally, such slow gasses facilitate suppression of electronics noise by allowing additional filtering and sample-averaging. For the purpose of the sensitivity estimates reported in Sec. 2.6.1, we use the negativeion drift gas 94:6 Ar: $\mathrm{CS}_{2}$ since gain data near 1 atm in a GEM have been published for this particular mixture [36]. Drift velocity and diffusion data have been published for other Ar: $\mathrm{CS}_{2}$ mixtures [40] allowing the use of Blanc's law [8, Ch. 2] to infer the drift velocity for the $94: 6$ ratio. The drift velocity of ions is proportional to $E / P$ ( $E=$ electric field; $P=$ pressure) up to fields far beyond what we would consider. Ion diffusion is usually independent of $P$. Drift and diffusion of $\mathrm{CS}_{2}$ ions have both been found to remain thermal near 1 atm in helium [32].

Extrapolation of the $0.92 \mathrm{~atm}$ (700 Torr) $\mathrm{Ar}: \mathrm{CS}_{2}$ data in [36] to $3 \mathrm{~atm}$ indicates that a single GEM layer would not provide adequate gain for photoelectron tracking. The extrapolation involves using a simplified (1-dimensional Gaussian) model of the field in a GEM hole and integrating the first Townsend coefficient, $\alpha$, to determine the gain. The form $\alpha=p A \exp (-B p / E)[60]$ is assumed with $A$ and $B$ fit from the 700 Torr data. Based on field calculations and other data, we assume an electron transparency (the fraction of primary electrons entering the avalanche region) of 0.8 . Published values for $A$ and $B$ are not available for $\mathrm{Ar}: \mathrm{CS}_{2}$ mixtures but we verified the accuracy of the model with a common electron drift gas $\left(\mathrm{Ar}+10 \% \mathrm{CH}_{4}\right.$ at 600 Torr). Generally, we find a net GEM gain of at least 2000 is needed to produce track images above the noise threshold. For an $\mathrm{Ar}: \mathrm{CS}_{2}$ mixture at $3 \mathrm{~atm}$, a stack of three GEMs would most likely be needed to exceed a net gain of 2000 . 
Table 1 Quantities influencing photoelectron track quality.

\begin{tabular}{lc|cc}
\hline Gas & $\mathrm{Ne}+8.3 \% \mathrm{CO}_{2}+8.3 \% \mathrm{CS}_{2}$ & $\mathrm{Ar}+6 \% \mathrm{CS}_{2}$ \\
Pressure & $0.63 \mathrm{~atm}$ & $\mathrm{~atm}$ \\
\hline Average drift distance & $0.4 \mathrm{~cm}$ & \multicolumn{2}{|c}{$5 \mathrm{~cm}$} \\
Pixel size & $132 \mu \mathrm{m}$ & \multicolumn{2}{|c}{1.2} \\
Drift diffusion (pixcls) & 0.24 & $20 \mathrm{kcV}$ & $50 \mathrm{keV}$ \\
\hline Photoclectron encrgy & $5.5 \mathrm{keV}$ & 17 & 81 \\
Photoclectron CSDA $^{a}$ range (pixels) & 13 & 0.058 & 0.13 \\
$\lambda_{e}{ }^{b}$ (pixcls) & 0.22 & $6.1^{\circ}$ & $2.2^{\circ}$ \\
Elastic scattering angle $^{c}$ & $5.5^{\circ}$ & & \\
\hline
\end{tabular}

${ }^{a}$ CSDA - Continuous Slowing Down Approximation for ionization encrgy loss.

${ }^{b} \lambda_{c}$-photoclectron elastic scattering mean free path [43].

c A small angle approximation is used here only for comparison purposes (sec text for discussion).

In the design shown in Fig. 7, the aperture of the detector is $10 \times 10 \mathrm{~cm}^{2}$. The depth is $10 \mathrm{~cm}$, which results in an absorption probability of $25 \%$ at $20 \mathrm{keV}$. For a fixed GEM area, it is more advantageous to further improve effective area by increasing the aperture rather than increasing depth. It may be advantageous to increase the pressure, but we limited it to $3 \mathrm{~atm}$ because, at that level, we believe it poses a relatively simple engineering problem.

Table 1 compares quantities influencing the quality of photoelectron tracking in two gas mixtures at $5.5 \mathrm{keV}$ (where we have polarization measurements) and 20 $50 \mathrm{keV}$ (where we do not yet have polarization measurements). ${ }^{2}$ The "elastic scattering angle" is based on a least-squares fit of the central portion of the full crosssections from [43] to a Gaussian distribution. The best-fit single-scatter "sigma" is multiplied by $\sqrt{N}$, where $N$ is the mean number of scatters occurring over the pixel size (at a fixed energy). This approximation neglects very large single-scatters which are likely to occur at some points along the track.

Although the distance between elastic collisions is much shorter in gas at $3 \mathrm{~atm}$, this is offset by higher energy electrons having a longer range and smaller singlescattering angles. Modulation data using the Ne: $\mathrm{CO}_{2}: \mathrm{CS}_{2}$ gas is shown in Fig. 4.

\subsection{Detector Unit}

The detector shown in Fig. 7 would be operated as a sealed proportional counter inside a pressure chamber. This simplifies the construction and operation of the detector. Prior to operation, the chamber can be evacuated with a turbomolecular pump but the sealed operation requires the use of low out-gassing materials and techniques similar to vacuum practices for the $10^{-9}$ Torr range in order for the gas diffusion and drift velocity to remain stable over days or weeks required for long-duration balloon flights.

\footnotetext{
${ }^{2}$ For neon, the $\mathrm{K}$-cdge is $0.87 \mathrm{keV}$. Thus the photoclectron energy is reduced from the $6.4 \mathrm{keV} \mathrm{X}$-ray energy by this amount in the neon-based mixture. For the sake of simplicity, we neglect the K-edges of the other elements in this comparison.
} 
Sealed operations also require vacuum and pressure transducers, high-voltage feedthroughs for biasing the drift electrode and GEM, and low-voltage feedthroughs for signals and ASIC power. A $125 \mu \mathrm{m}$ aluminum window with a metal strongback allows most $(>80 \%$ ) incident $\mathrm{X}$-ray photons above $15 \mathrm{keV}$ to enter the active region between the drift electrode and the GEM stack. Inside the chamber, field-shaping electrodes surround the active region to eliminate false modulation arising from driftfield non-uniformity, creating an electric field that is uniform in magnitude to $\sim 1 \%$ and in direction to a few degrees. To minimize out-gassing material, only the ASICs are mounted in the chamber.

A stack of GEMs with $140 \mu \mathrm{m}$ pitch and $10 \times 10 \mathrm{~cm}^{2}$ area from either SciEnergy or Tech-Etch can be used in the flight unit based on on-going evaluations.

The readout strips can be fabricated on LCP similar to the $3 \times 3 \mathrm{~cm}^{2}$ strips built for GRBP and the $2 \times 5 \mathrm{~cm}^{2}$ strips for GEMS-XPI. Strips are typically placed $1 \mathrm{~mm}$ beneath the GEM stack.

The detector module consists of 640 readout strips with a pitch of $150 \mu \mathrm{m}$. The strips can be read out by 5 ASICs, with each strip individually instrumented similar to the GEMS-XPI design (see Fig. 5, right).

The higher the drift field, the lower the diffusion and faster the ions. $10 \mathrm{kV}$ is the highest drift voltage that is economical and easily insulated. This would provide a drift velocity of about $600 \mathrm{~cm} / \mathrm{s}$ with Ar: $\mathrm{CS}_{2} 94: 6$ at $3 \mathrm{~atm}$. For this drift velocity, strips would be readout at a rate of $40.3 \mathrm{kHz}$ to make time bins equivalent to the chosen spatial pitch of $150 \mu \mathrm{m}$.

The Instrument Control Electronics (ICE) include a low-voltage power supply (LVPS), high-voltage power supply (HVPS), engineering data system (EDS), and command and data handling (C\&DH). The balloon payload could reuse the GRBP electronics design described below.

The FPGA on the GRBP command and data handling (C\&DH) board includes both the interface with the ICE, an instrument data processing function, and the balloon RS422 interface.

Two controllable high-voltage supplies are used to bias the detector: a negative $8 \mathrm{kV}$ supply for the drift electrode to allow adjustment of the drift velocity and a negative $2 \mathrm{kV}$ supply with a voltage-divider circuit to control the GEM electrodes relative to the strips. The negative $8 \mathrm{kV}$ supply will also provide the voltages for the field cage around each detector. The C\&DH board has a $17 \times 17 \mathrm{~cm}^{2}$ footprint. The electronics enclosure that houses the four boards and constitutes the instrument electronics box. For a balloon-borne instrument, special care must be taken to avoid high-voltage breakdown at the partial pressures at balloon altitudes. Consequently, the IXPS electronics box would be enclosed in a pressurized chamber at 1 atm that is mounted directly to the TPC pressurized enclosure so that no HV lines will be exposed to the partial pressure.

The overall parameters of the IXPS instrument are shown in Table 2 . 
Table 2 Estimated instrument payload parameters.

\begin{tabular}{ll}
\hline Mass & $20 \mathrm{~kg}$ \\
\hline Volume & \\
$\quad$ Active & $10 \mathrm{~cm} \times 10 \mathrm{~cm} \times 10 \mathrm{~cm}=1000 \mathrm{~cm}^{3}$ \\
Detector and clectronics & $30 \mathrm{~cm} \times 50 \mathrm{~cm} \times 25 \mathrm{~cm}$ \\
Collimators & $10 \mathrm{~cm} \times 10 \mathrm{~cm}$ front and rear separated by $100 \mathrm{~cm}$. \\
\hline Power & 30 watts \\
\hline Data Rate & 2 Gbytes per X-class flare \\
\hline
\end{tabular}

\subsection{Imaging Capability}

Imaging X-ray polarimetry with an angular resolution of better than $10 \operatorname{arcsec} c a n$ determine the polarization of different sources in a flare (e.g., the separate footpoints and loop tops). This capability can be achieved by measuring the spatial Fourier components of the X-ray flare as is done with RHESSI. RHESSI measures hundreds of Fourier components by using a bi-grid modulation collimator placed above each of its nine germanium detectors and spinning the whole spacecraft about a Sun-pointed axis. The collimators have grids with different slit pitches, allowing a range of source sizes between 2 and 180 arcseconds to be covered. No spatial information is required of the germanium detectors in this design. Although such a technique could be used for imaging X-ray polarimetry on a future space mission (assuming an electron gas and fast electronics), rotating a balloon-borne instrument about the direction to the Sun is not feasible and rotating individual bi-grid collimators is not easy. Consequently, for initial balloon flights, a modified, non-rotating approach can be used that takes advantage of the one-dimensional spatial information in the TPC. This approach uses stationary bi-grid collimators with grid pitches and orientations chosen to generate Moiré fringes in the detector plane. Judicial choices for these parameters allow the Moiré fringes to be oriented at any desired angle relative to the grid-slat direction (which is perpendicular to the direction of the sampled Fourier component) (Fig. 9). The phase and amplitude of these Moiré fringes are related to the corresponding spatial Fourier components of the source region, but measuring them does require a detector with spatial information in one dimension. A TPC is suitable for this purpose.

The statistics for a typical M-class flare detected with IXPS do not warrant the measurement of hundreds of Fourier components that RHESSI is capable of doing. However, measurement of just a few judiciously chosen Fourier components can provide enough information to separate the different flare sources, especially if contemporaneous images of the flare are available in the same energy range from a different instrument such as RHESSI. Consequently, we envision measuring just four Fourier components covering several different orientations about the Earth-Sun line but with the same spatial period ( 10 arcseconds), chosen to cover the expected source dimensions and separations.

The TPC can measure the amplitude and phase of a Moiré fringe by determining the position of the photoelectric interaction for each measured electron track in 


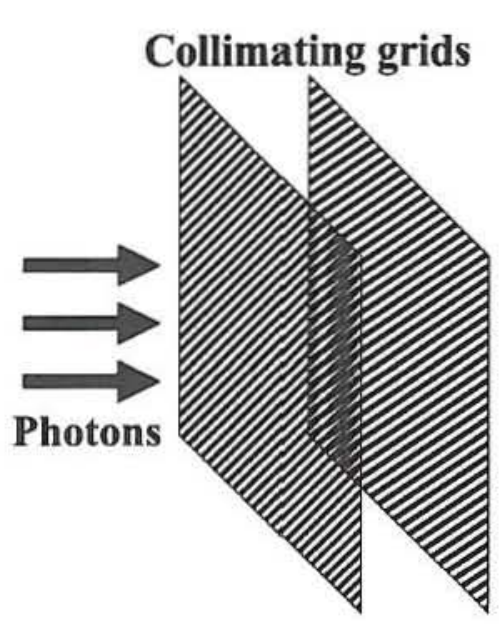

Fig. $9 \mathrm{X}$-rays passing through a bi-grid collimator producc a Moire pattern at the detector. The orientation of the pattern is controlled by two parameters that affect fringing in orthogonal directions- the difference in the pitch of the two grids and the difference in the roll angle of the slits in the two grids. Judicial choice of these two parameters (here $8 \%$ pitch difference and $5^{\circ}$ roll angle difference) results in Moirć fringes at the desired angle relative to the mean gridslit roll angle (here $\sim 45^{\circ}$ ). In principle, only one period of the Moiré pattern is needed to determine the phase and amplitude required to characterize a single spatial Fourier component of the source.

the plane perpendicular to the direction to the Sun (X-Y plane in Fig. 6 and 7). In the drift direction ( $\mathrm{X}$ axis), the position of the photoelectric interaction cannot be determined accurately, although some information can be obtained from the increased track dispersion with drift distance. However, in the orthogonal direction (Y axis), the position of the photoelectric interaction is determined with sub-mm accuracy by identifying the strip at the start of the electron track. To measure a Fourier component in any direction, the relative pitch and orientation of the slits of the front and rear grids are arranged to produce Moiré fringes that are aligned perpendicular to this spatially sensitive direction-see Fig. 9.

The detailed design of the collimator system can be based on an analysis of RHESSI images for typical M- and X-class flares to determine the optimum Fourier components to measure. Grids with a pitch of $100 \mu \mathrm{m}$ (near that of the third finest RHESSI grids) separated by $1 \mathrm{~m}$ (cf. $1.55 \mathrm{~m}$ for RHESSI) would give a FWHM angular resolution of $\sim 10$ arcsec. This would be adequate for generating the required Moiré fringes and separating the different flare sources of interest for polarimetry. We envision four grid pairs, each one covering a separate $2.5 \times 10 \mathrm{~cm}^{2}$ section of the TPC. The relative slit pitch and orientation of the two grids of each pair can be specified to generate just one period of the Moiré fringe in that section as needed to determine uniquely the phase and amplitude of the corresponding spatial Fourier component of the X-ray source on the Sun.

\subsection{Sensitivity Estimation Techniques}

In this section, we outline the techniques used for the calculation of the polarization sensitivity of the flight instrument. The analysis shows more than an order-ofmagnitude increase in sensitivity to flare polarization over existing instruments. 
To estimate the detector sensitivity to polarization, we have written detector simulation and track-reconstruction code that predicts the sensitivities of various polarimeter configurations. The software reproduces the measured modulation for $4.5 \mathrm{keV} \mathrm{X}$ rays [6], and produces results in agreement with similar simulations [4]. The code simulates photoelectrons emitted with a $\sin ^{2} \theta \cos ^{2} \phi$ probability distribution and an isotropically distributed Auger electron. For each photoelectric interaction, the two electrons propagate through the detector gas according to a single scattering model developed for electron microscopy [24]. Ionization electrons produced along the track are propagated with diffusion through the drift region to the GEM. Each ionization electron produces an avalanche with proportional counter statistics. The avalanche electrons are each assigned to a virtual detector pixel. The photoelectron track reconstruction algorithm follows the prescription described in [7]. The simulation predicts a modulation factor of $>50 \%$ across the $10 \mathrm{~cm}$ drift region (Fig. 8).

Because the measured modulation is a positive-definite quantity, even an unpolarized source can give an apparent positive polarization. The sensitivity of a polarimeter is therefore expressed as the minimum detectable polarization (MDP), a polarization result that is statistically unlikely to arise from an unpolarized source. The MDP is a function of instrumental properties, the source strength, $S$, and the observing time, $t$. At the $99 \%$ confidence level,

$$
M D P=\frac{4.29}{\varepsilon \mu S A}\left(\frac{\varepsilon S A+B}{t}\right)^{1 / 2}
$$

where $\varepsilon=$ quantum efficiency, $\mu=$ modulation factor, $A=$ collecting area, and $B=$ background rate [56].

In the strong source approximation valid for bright solar flares detected by IXPS, $S \gg B$, the MDP scales with instrument parameters as $1 / \mu \sqrt{\varepsilon A}$. The quantity $\mu \sqrt{\varepsilon A}$ may be used as an instrumental figure-of-merit dependent on detector dimensions, gas, pressure, etc.

To calculate MDP for an instrument, the modulation factor $\mu(E)$ is weighted by the number of events per unit energy as determined from the source spectrum, the detector quantum efficiency, and the collimator transmission. Finally, the broadband modulation factor $(\mu)$ and the expected number of source counts are used to calculate the MDP.

\subsubsection{Sensitivity Estimate}

To characterize the instrument sensitivity, we use the spectra measured with RHESSI for an M1.2 flare that occurred on 2002 April 15 [49] and an X4.8 flare that occurred on 2002 July 23 [31]. We use a $200 \mathrm{~s}$ integration time when the flare was brightest in each case. A longer integration time would be possible for longer duration flares resulting in commensurately better (i.e., lower) MDP values.

The flux for the M1.2 flare was $\sim(E /(28.4 \mathrm{keV}))^{-6.2}$ photons s${ }^{-1} \mathrm{~cm}^{-2} \mathrm{keV}^{-1}$ with photon energy $E$ in keV. For the X4.8 flare, the flux was $\sim(E /(66.2 \mathrm{keV}))^{-6.4}$ for $E<20 \mathrm{keV}$ and $\sim(E /(13.3 \mathrm{keV}))^{-7.7}$ for $E \geq 20 \mathrm{keV}$. These formulae are only provided for reference. The actual MDP calculation used log-log interpolation of tabulated data. 
To avoid modeling a flare, the flux is summed over all regions of a flare. This leads to a lower MDP estimate than actually would be found from imaging a particular region of a flare. Despite this, the likelihood of observing a significant polarization through imaging would be enhanced since the polarization percentage would not be diluted by regions with relatively unpolarized emission. For example, if most of the source strength were unpolarized and background small, the MDP estimate without imaging would scale as $\sim S^{-1 / 2}$ (Eq. 1) but the measured polarization percentage would degrade as $\sim S^{-1}$. When imaging a particular region, $S$ would be smaller but the measured polarization percentage would be mostly independent of $S$ (assuming sufficient angular resolution).

The effects of an X-ray bi-grid modulation collimator with an average transmission of $25 \%$ (equal slat and slit widths) and a window equivalent to $125 \mu \mathrm{m}$ of aluminum plus a strongback with $10 \%$ obscuration is included in the calculation. The assumed atmospheric path length at the balloon altitude was taken as $5 \mathrm{~g} \mathrm{~cm}^{-2}$ from Palestine, Texas, and $10 \mathrm{~g} \mathrm{~cm}^{-2}$ in Antarctica with the Sun's elevation angle of $45^{\circ}$ and $30^{\circ}$, respectively. The MDP values obtained in this way are summarized in Table 3. They show that with a single $10 \times 10 \times 10 \mathrm{~cm}^{3}$ TPC the instrument has high polarization sensitivity to the X-class flare from both Palestine and Antarctica, whereas any polarization of an M1 flare could only be determined from Palestine with an MDP of $13 \%$. We expect that polarization of an M5 flare would be detectable at the $\sim 15 \%$ level from Antarctica if it lasted for $>10 \mathrm{~min}$. If the X-ray flare rate matches the average of the previous three cycles, then we can expect to see at least one such flare or larger during a >10-day Antarctic balloon flight. Likewise, for a 6-hour balloon flight, we can expect to see a flare of class at least M1 $\sim 20 \%$ of the time.

The detector deadtime in recording an event is the digitizer readout time of $\sim 100 \mu$ s. An X1 flare will produce about $10^{4}$ events per second in each detector, so readout deadtime will be $\sim 50 \%$. However, it will be precisely measured allowing an accurate determination of the flux of incident photons from the rate of events that are actually recorded. Track confusion caused by photoelectrons produced throughout the volume should not be significant at this rate. It is only a problem if two tracks occupy the same imaging area during the readout time. Since a typical track will be $\sim 1 \mathrm{~cm}$ long, the relevant area is only $\sim 1 \mathrm{~cm}^{2}$ as compared to the $100 \mathrm{~cm}^{2}$ total area. Thus, the rate of tracks that will be confused in the readout will be down by this ratio of 100 . However, tracks from proton background can be much longer, preventing collection of simultaneous X-rays while charge from these events is collected in the GEM.

To estimate the deadtime from charged particles, we assume that the maximum deadtime per track is $17 \mathrm{~ms}$ - the time for charge to drift from the drift electrode to the GEM. Based on the cosmic-ray background simulator results presented by [37], we estimate a count rate of $\sim 60 \mathrm{~s}^{-1}$ passing through the detector in Palestine and $\sim 200 \mathrm{~s}^{-1}$ in Antarctica. The deadtime from particles is small in Palestine but not for M-class flares in Antarctica where the X-ray rate is low and the particle rate is high. (For a mission to Antarctica, use of an electron gas such as an Argon-DME mixture with fast read-out electronics may be justified despite the additional cost and power consumption.) For the MDP calculations, we assumed that all of these particle events 
Table 3 Estimated MDP values at energies above $\sim 20 \mathrm{keV}$ for $200 \mathrm{~s}$ at the peak of two flares with different GOES class for the proposed single TPC polarimeter on balloon flights from Palestine (Texas) and Antarctica, and for a possible future instrument in space with 12 times the sensitive volume using 4 TPCs each with $3 \times$ the volume. To avoid modeling a flare, the flux is summed over all regions of a flare. This gives a more optimistic MDP estimate than would actually be obtained with grids. Grid-based imaging would provide separate MDPs for different regions of the flare. Although each each region would have a higher MDP, grid-based imaging would enhance the likelihood of observing higher polarization regions of the flare since the polarization percentage would be less diluted by low polarization regions.

\begin{tabular}{|c|c|c|c|c|c|}
\hline & \multicolumn{2}{|c|}{ Palestine, TX } & \multicolumn{2}{|c|}{ Antarctica } & Space \\
\hline Mean solar elev. angle & \multicolumn{2}{|c|}{$45^{\circ}$} & \multicolumn{2}{|c|}{$30^{\circ}$} & - \\
\hline Atmos. path length & \multicolumn{2}{|c|}{$5 \mathrm{gcm}^{-2}$} & \multicolumn{2}{|c|}{$10 \mathrm{gcm}^{2}$} & - \\
\hline TPC volume & \multicolumn{2}{|c|}{$10^{3} \mathrm{~cm}^{3}$} & \multicolumn{2}{|c|}{$10^{3} \mathrm{~cm}^{3}$} & $12 \times 10^{3} \mathrm{~cm}^{3}$ \\
\hline Grids & No & Yes & No & Yes & Yes \\
\hline GOES M1.2 flare & $13 \%$ & $23 \%$ & - & - & $2 \%$ \\
\hline GOES X 4.8 flare & $3 \%$ & $4 \%$ & $6 \%$ & $11 \%$ & $2 \%$ \\
\hline
\end{tabular}

can be identified and rejected using the various techniques discussed in Section 2.2.1, and will not degrade the analysis of photoelectron events.

For larger flares giving an even higher rate, some track confusion and uncertainty in the deposited energy estimates can be expected. In that case, a movable shutter system can be devised to restrict the aperture to a narrow $(\sim 1 \mathrm{~cm}$ wide) strip across the detector perpendicular to the strips. This would reduce the counting rate by a factor of 10 while still allowing the amplitude and phases of the Moiré fringes to be accurately measured. This shutter system would restrict the sensitive area at all energies equally. Such a shutter system will be necessary on a satellite instrument but is probably not justified on a long-duration balloon flight since the probability of seeing such large flares that only occur a few times per solar cycle is so low.

\subsubsection{Testing and Calibration}

TPC systematics must be determined with sufficient accuracy to allow an MDP of $2 \%$ or less for a sufficiently large flare. We estimate modulations of approximately $50 \%$, so this requirement translates to understanding the response to unmodulated X-rays to $1 \%$ at the $99 \%$ level of confidence.

Initial testing can be performed in an established configuration used for testing 2$10 \mathrm{keV}$ polarimeters. The polarimeter can be tested with a $\mathrm{Ne} / \mathrm{CO}_{2} / \mathrm{CS}_{2}$ mixture at a pressure of $\sim 1 \mathrm{~atm}$ and with an existing $100 \%$ polarized 6.4 and $6 \mathrm{keV} \mathrm{X-ray} \mathrm{source.}$ For tests at these lower energies, a beryllium window is used rather than aluminum.

The modulation must be measured as a function of energy, drift distance, and pressure in the expected operating range. Measuring the response to X-rays from radioisotope decay is the best end-to-end calibration in the laboratory. The unmodulated response can be measured with unpolarized $60 \mathrm{keV} \mathrm{X-rays} \mathrm{from} \mathrm{a} 1 \mathrm{mCi}{ }^{241} \mathrm{Am}$ source. This is bright enough to allow a search for calibration variations on timescales from minutes to days. Lower energy X-ray sources such as ${ }^{109} \mathrm{Cd}(22 \mathrm{keV})$ can be used to span the relevant energy range of $20-50 \mathrm{keV}^{3}{ }^{3} \mathrm{The}^{241} \mathrm{Am}$ results can

${ }^{3} 0.1 \mathrm{mCi}$ at $22 \mathrm{keV}$ should have comparable detection rate to $1 \mathrm{mCi}$ at $60 \mathrm{keV}$. 
be compared to techniques that directly measure the drift velocity with a UV laser calibration system [1].

Polarized X-rays from $20-50 \mathrm{keV}$ can be obtained from a variety of terrestrial sources: from a synchrotron such as the NSLS at Brookhaven National Laboratory, from Compton scattering of unpolarized X-rays at $90^{\circ}$ of incidence [42], or from Bragg scattering (reflection) of unpolarized X-rays through $90^{\circ}$ off synthetic diamond [45].

\subsubsection{Crab Sensitivity}

We estimate a count rate of 0.8 counts $\mathrm{s}^{-1}$ from the Crab nebula plus pulsar for the $10 \times 10 \times 10 \mathrm{~cm}^{3}$ detector shown in Fig. 7 filled with $3 \mathrm{~atm}$ of $\mathrm{Ar}: \mathrm{CS}_{2}$ 94:6 and with no modulation collimator. We assume an atmospheric overburden of $5 \mathrm{~g} \mathrm{~cm}^{-2}$, a Crab spectrum of $10 E^{-2.1}$ photons $\mathrm{cm}^{-2} \mathrm{~s}^{-1} \mathrm{keV}^{-1}$ [59], and integrate the resulting count rate from $15-50 \mathrm{keV}$. Under the assumption that the source rate is large compared to the background, and that the modulation factor $(\mu)$ is 0.5 across the energy band, a polarization could be detected with $99 \%$ confidence at $>19 \% \times \sqrt{t / 4 \mathrm{hrs}}$ where $t$ is the net on-source observing time. The Crab nebula is known to be polarized at the level of $19 \%$ at $5.2 \mathrm{keV}$ [58] and appears to increase to over $40 \%$ at higher energies [13]. Thus, such an instrument would be able to provide a useful measure of the Crab polarization.

Note that the background of relevance in the calculation of the MDP is the rate of tracks that appear to be photoelectrons. It does not include events that can be eliminated as being from other origins as discussed above. Even if this residual background rate is equal to the source rate, polarization from the Crab nebula could still be detected in a 4-hour or greater exposure. The pulsar may dilute the total polarization signal, but as the pulsar contributes a modest fraction of the overall counts $(\sim 15 \%$ in our energy band), the conclusion remains that the proposed instrument could measure the polarization in a band with peak sensitivity between 20 and $30 \mathrm{keV}$.

Acknowledgements This work was supported by NASA through the Heliophysics Low Cost Access to Space program of the Rescarch Opportunities in Space and Earth Sciences (ROSES). Several individuals have provided technical assistance or advice for this project. We thank Bob Baker and Ken Simms for custom electronics. Michael Dion assisted especially with the cleaning and stretching of GEMs. Jeff Martoff provided useful advice and ideas particularly concerning detector gas. Zach Prieskorn's work with a similar detector provided a uscful baseline for testing and troubleshooting various problems. Phil Kaaret suggested polarization through Compton scattering and provided references. We thank Toru Tamagawa for help communicating with Japancse suppliers. Finally, we thank Zhong Zhong for assistance concerning the use of synchrotron radiation for $20-50 \mathrm{kcV}$ polarized X-rays.

\section{References}

1. Anderhub, H., Devereux, M.J., Seiler, P.G.: On a new method for testing and calibrating ionizing particle detectors. Nucl. Instrum. and Methods A 166, 581-582 (1979)

2. Bai, T., Ramaty, R.: Backscatter, anisotropy, and polarization of solar hard X-rays. Astrophys. J. 219, 705 (1978)

3. Bellazzini, R., Costa, E., Matt, G., Tagliaferri, G. (cds.): X-ray Polarimetry: A New Window in Astrophysics. Cambridge Contemporary Astrophysics. Cambridge University Press (2010) 
4. Bellazzini, R., et al: Reading a GEM with a VLSI pixel ASIC used as a direct charge collecting anode. Nucl. Instrum. and Methods A 535, 477 (2004)

5. Black, J.K., et al.: The imaging X-ray detector for Lobster-ISS. Nucl. Instrum. and Methods A 513, $123(2003)$

6. Black, J.K., et al.: X-ray polarimetry with an active-matrix pixel proportional counter. Nucl. Instrum. and Mcthods A 513, 639 (2003)

7. Black, J.K., et al.: X-ray polarimetry with a micropattern TPC. Nucl. Instrum. and Methods A 581, 755 (2007)

8. Blum, W., Rolandi, L.: Particle Detection with Drift Chambers. Springer-Verlag (1993)

9. Boggs, S.E., Coburn, W., Kalcmci, E.: Backscatter, anisotropy, and polarization of solar hard X-rays. Astrophys. J. 638, 1129 (2006)

10. Bogomolov, A.V., ct al.: Polarization of hard X-rays in October-November, 2003 solar flares observed onboard CORONAS-F satellite. In: A.V. Stepanov, E.E. Benevolenskaya, A.G. Kosovichev (eds.) Multi-Wavelength Investigations of Solar Activity, no. 223 in IAU Symposium, p. 447. Cambridge University Press (2004)

11. Brown, J.C.: The directivity and polarisation of thick target X-ray bremsstrahlung from solar flares. Sol. Phys. 26, 441 (1972)

12. Brown, J.C., McClymont, A.N., McLean, I.S.: Interpretation of solar hard X-ray burst polarisation measurements. Nature 247, 448 (1974)

13. Dean, A.J., et al.: Polarized gamma-ray emission from the Crab. Science 321, 1183 (2008)

14. Emslie, A.G., Bradsher, H.L., McConnell, M.L.: Hard X-ray polarization from non-vertical solar flare loops. Astrophys. J, 674, 570-575 (2008). doi:10.1086/524983

15. Emslic, A.G., Brown, J.C.: The polarization and directivity of solar-flare hard X-ray bremsstrahlung from a thermal source. Astrophys. J. 237, 1015-1023 (1980). doi:10.1086/157947

16. Emslie, A.G., Vlahos, L.: Radiation signatures from a locally energized flaring loop. Astrophys. J. 242, 359-373 (1980), doi: $10.1086 / 158469$

17. Emslie, A.G., ct al.: RHESSI hard X-ray imaging spectroscopy of the large gamma-ray flare of 2002 July 23. Astrophys, J. Lett. 595, L107-L110 (2003). doi:10.1086/378931

18. Forot, M., Laurent, P., Grenier, I.A., Gouiffes, C., Lebrun, F.: Polarization of the Crab Pulsar and Nebula as observed by the INTEGRAL/IBIS telescope. Astrophys. J. Lett. 688, L29-L32 (2008). doi:10.1086/593974

19. Gluckstem, R.L., Hull Jr., M.H.: Polarization dependence of the integrated bremsstrahlung cross section. Phys. Rev, 90, 1030-1035 (1953)

20. Haug, E.: Polarization of hard X-rays from solar flares. Sol. Phys. 25, 425-434 (1972). doi:10.1007/ BF00192340

21. Hénoux, J.C.: Anisotropy and polarization of solar X-ray bursts. Sol. Phys, 42, 219-233 (1975). doi:10.1007/BF00153298

22. Hill, J.E., et al.: A burst chasing X-ray polarimeter. In: O.H.W. Siegmund (cd.) UV, X-Ray, and Gamma-Ray Space Instrumentation for Astronomy XV, Proc. Soc. Photo-Opt. Instrum. Eng., vol. 6686 , p. $66860 \mathrm{Y}(2007)$

23. Holman, G.D., et al.: Electron bremsstrahlung hard $\mathrm{X}$-ray spectra, electron distributions, and energetics in the 2002 July 23 solar flare. Astrophys. J. Lett. 595, L97-L10I (2003). doi:10.1086/378488

24. Joy, D.C.: Monte Carlo Modeling for Electron Microscopy and Microanalysis. Oxford Series in Optical and Imaging Sciences. Oxford University Press, Inc. (1995)

25. Kontar, E.P., Brown, J.C.: Stercoscopic clectron spectroscopy of solar hard X-ray flares with a single spacccraft. Astrophys. J. Lett. 653, L149-L152 (2006). doi:10.1086/510586

26. Kontar, E.P., Jeffrcy, N.L.S.: Positions and sizes of X-ray solar flare sources. Astron. Astrophys. 513, $\mathrm{L} 2+(2010)$. doi:10.1051/0004-6361/201014066

27. Langer, S.H., Petrosian, V.: Impulsive solar X-ray bursts. III - Polarization, directivity, and spectrum of the refiected and total bremsstrahlung radiation from a beam of electrons directed toward the photosphere. Astrophys. J. Lett. 215, 666-676 (1977). doi:10.1086/155400

28. Leach, J., Petrosian, V:: The impulsive phase of solar flares. II - Characteristics of the hard X-rays. Astrophys. J. 269, 715-727 (1983). doi:10.1086/161081

29. Leach, J., Petrosian, V., Emslic, A.G.: The interpretation of hard X-ray polarization mcasurements in solar flares. Sol. Phys, 96, 331-337 (1985). doi:10.1007/BF00149688

30. Lin, R.P., et al.: The Reuven Ramaty High-Energy Solar Spectroscopic Imager (RHESSI). Sol. Phys. 210, 3-32 (2002). doi:10.1023/A:1022428818870

31. Lin, R.P., et al. RHESSI observations of particle acceleration and energy release in an intense solar gamma-ray line flare. Astrophys. J. Lett. 595, L69-L76 (2003), doi:10.1086/378932 
32. Martoff, C., Ayad, R., Katz-Hyman, M., Bonvicini, G., Schreiner, A.: Negative ion drift and diffusion in a TPC near 1 bar. Nucl. Instrum. and Methods A 555(1-2), 55-58 (2005). doi:10.1016/j.nima. 2005.08.103

33. McConnell, M.L., et al.: RHESSI as a hard X-ray polarimeter. Sol. Phys. 210, 125-142 (2002). doi:10.1023/A:1022413708738

34. McConnell, M.L., et al.: RHESSI solar flare polarization measurements in the $20-100 \mathrm{kcV}$ energy range. In: American Astronomical Socicty Mecting 210, \#93.01, Bulletin of the American Astronomical Society, vol. 38, p. 211 (2007)

35. McConnell, M.L., et al.: GRAPE: a balloon-borne gamma-ray polarimeter. In: O.H. Siegmund (ed.) UV, X-Ray, and Gamma-Ray Space Instrumentation for Astronomy XVI, Soc. Photo-Opt. Instrum. Eng. (SPIE) Conference Series, vol. 7435, p. 74350J (2009). doi:10.1117/12.826407

36. Miyamoto, J., et al.: GEM operation in negative ion drift gas mixtures. Nucl. Instrum. and Methods A 526(3), 409-412 (2004). doi:10.1016/j.nima,2004.02.018

37. Mizuno, T., et al.: Geant4 based cosmic-ray background simulator for balloon experiments. In: Nuclear Science Symposium Conference Record, 2001 IEEE, vol. 1, pp. 442-446 (2001)

38. Murphy, R.J., et al.: Physical implications of RHESSI neutron-capture line measurements. Astrophys. J. Lett. 595, L93-L96 (2003). doi:10.1086/378175

39. Novick, $\mathrm{R}$, et al.: Detection of X-ray polarization of the Crab Nebula. Astrophys. J. Lett. 174, L1+ (1972). doi:10.1086/180938

40. Ohnuki, T., Snowden-Ifft, D.P., Martoff, C.J.: Measurement of carbon disulfide anion diffusion in a TPC. Nucl. Instrum. and Methods A 463(1-2), 142-148 (2001). doi:10.1016/S0168-9002(01)00222-4

41. Pacciani, L., et al.: Sensitivity of a photoclectric X-ray polarimeter for astronomy: the impact of the gas mixture and pressure. In: S. Fineschi (ed.) Polarimetry in Astronomy, Proc. Soc. Photo-Opt. Instrum. Eng., vol. 4843, pp. $394-405$ (2003). doi; 10.1117/12.459275

42. Sakurai, H., Noma, M., Niizcki, H.: A hard X-ray polarimeter utilizing Compton scattering. In: EUV Optics for Astronomy, Microscopy, Polarimetry, and Projection Lithography, Proc. Soc. Photo-Opt. Instrum. Eng., vol. 1343, pp. 512-518 (1990)

43. Salvat, F., Jablonski, A.: NIST Standard Reference Database 64-NIST Electron Elastic-Scattering Cross-Section Database Version 3.1. National Institue of Standards and Technology (NIST) (2003). http://www.nist.gov/srd/nist64.cfm. Accessed 18 October 2010

44. Shih, A.Y, et al:: The Gamma-Ray Imager/Polarimeter For Solar Flares (GRIPS). In: AAS/Solar Physics Division Meeting 40, \#18.10, Bulletin of the American Astronomical Society, vol. 41, p. 846 (2009)

45. Shvyd'ko, Y.V., et al.: High-reflectivity high-resolution X-ray crystal optics with diamonds. Nat. Phys. 6, 196-199 (2010). doi:10.1038/NPHYS1506

46. Soffitta, P., et al.: Proportional counters for the Stellar X-Ray Polarimeter with a wedge and strip cathode pattern readout system. Nucl. Instrum. and Methods A 414, 218-232 (1998). doi:10.1016/ S0168-9002(98)00572-5

47. Soffitta, P., et al.: A set of X-ray polarimeters for the New Hard X-ray Imaging and Polarimetric Mission. In: M. Arnaud, S.S. Murray, T. Takahashi (eds.) Space Telescopes and Instumentation 2010: Ultraviolet to Gamma Ray, Proc. Soc. Photo-Opt. Instrum. Eng., vol. 7732, p. 77321A (2010). doi: $10.1117 / 12.856434$

48. Suarez-Garcia, E., et al.: X-ray polarization of solar flares measured with RHESSI. Sol. Phys. 239, 149-172 (2006). doi:10.1007/s11207-006-0268-1

49. Sui, L., Holman, G.D., Dennis, B.R.: Determination of low-energy cutoffs and total energy of nonthermal electrons in a solar flare on 2002 April 15. Astrophys. J. 626, 1102-1109 (2005). doi: $10.1086 / 430086$

50. Swank, J., Kallman, T., Jahoda, K.: Gravity and Extreme Magnetism SMEX. In: 37th COSPAR Scientific Assembly, COSPAR, Plenary Meeting, vol. 37, p. 3102 (2008)

51. Tindo, I.P., et al.: On the polarization of the emission of X-ray solar flares. Sol. Phys. 14, 204-207 (1970). doi:10.1007/BF00240179

52. Tindo, L.P., et al.: New measurements of the polarization of X-ray solar flares. Sol. Phys. 24, 429-433 (1972). doi:10.1007/BF00153385

53. Tindo, I.P., et al.: Preliminary interpretation of the polarization measurements performed on 'Intercosmos-4' during three X-ray solar flares. Sol. Phys. 27, 426-435 (1972). doi:10.1007/ BF00153113

54. Tindo, I.P., et al.: Further polarization measurements of the solar flare X-ray emission. Sol. Phys. 32, $469-475$ (1973). doi:10.1007/BF00154959 
55. Tramiel, L.J., Novick, R., Chanan, G.A.: Polarization evidence for the isotropy of electrons responsible for the production of 5-20 keV X-rays in solar flares. Astrophys. J. 280, 440-447 (1984). doi: $10.1086 / 162010$

56. Weisskopf, M.C., Elsner, R.F., O'Dell, S.L.: On understanding the figures of merit for detection and measurement of X-ray polarization. In: M. Amaud, S.S. Murray, T. Takahashi (eds.) Space Telescopes and Instumentation 2010: Ultraviolet to Gamma Ray, Proc. Soc. Photo-Opt. Instrum. Eng., vol. 7732, p. 77320 E (2010), doi: $10.1117 / 12.857357$

57. Weisskopf, M.C., et al.: Measurement of the X-ray polarization of the Crab Nebula. Astrophys. J. Lett. 208, L125-L128 (1976). doi:10.1086/182247

58. Weisskopf, M.C., et al.: A precision measurement of the X-ray polarization of the Crab Nebula without pulsar contamination. Astrophys. J. Lett. 220, L117-L121 (1978). doi:10.1086/182648

59. Weisskopf, M.C., et al.: On calibrations using the Crab Nebula and models of the nebular X-ray emission. Astrophys. J. 713, 912-919 (2010). doi:10.1088/0004-637X/713/2/912

60. Zastawny, A.: Standardization of gas amplification description in proportional counters. Nucl. Instrum. and Methods A 385, 239-242 (1997)

61. Zhitnik, I.A., et al:: Polarization, temporal, and spectral parameters of solar flare hard X-rays as measured by the SPR-N instrument onboard the CORONAS-F satellite. Sol. Syst. Res. 40, 93-103 (2006). doi:10.1134/S003809460602002X 